(C) 2010 International Press

Adv. Theor. Math. Phys. 14 (2010) 541-561

\title{
Vector bundles on Sasakian
}

\section{manifolds}

\author{
Indranil Biswas $^{1}$ and Georg Schumacher ${ }^{2}$
}

${ }^{1}$ School of Mathematics, Tata Institute of Fundamental Research, Homi Bhabha Road, Bombay 400005, India

indranil@math.tifr.res.in

${ }^{2}$ Fachbereich Mathematik und Informatik, Philipps-Universität Marburg, Lahnberge, Hans-Meerwein-Strasse, D-35032 Marburg, Germany schumac@mathematik.uni-marburg.de

\begin{abstract}
We investigate the analog of holomorphic vector bundles in the context of Sasakian manifolds.
\end{abstract}

\section{Introduction}

Just as the contact manifolds serve as substitutes for symplectic manifolds in odd dimensions, the Sasakian manifolds are the odd-dimensional counterparts of Kähler manifolds. Although they are around for quite some time, in recent years they were extensively studied. This resurgence is substantially influenced by the recently found relevance of Sasakian manifolds in string theory. Boyer and Galicki published a series of papers investigating various differential geometric aspects of Sasakian manifolds (see [4] and references therein). Sasakian manifolds appeared in string theory through the work of Maldacena [8]; see the papers of J. Sparks and references therein for more details.

e-print archive: http://lanl.arXiv.org/abs/0809.3892 
Our aim here is to investigate the analog of holomorphic vector bundles in the context of Sasakian manifolds, reflecting the rich theory of holomorphic vector bundles on Kähler manifolds, more precisely, the theory of moduli for stable Sasakian holomorphic vector bundles. We show the existence of the moduli space in the category of reduced complex spaces, and construct a Kähler structure on it.

Necessary prerequisites include a Hodge theory related to both the Sasakian and the holomorphic aspects. We arrive at a notion of a HermiteEinstein connection. Every stable Sasakian holomorphic vector bundle possesses a unique such connection. The variation of the Hermite-Einstein structures in a holomorphic family is closely related to infinitesimal deformations of the Sasakian holomorphic structures. We introduce an intrinsic Kähler metric on the moduli space, and show that, up to a numerical constant, it is equal to the Chern form of a determinant line bundle equipped with a Quillen metric.

\section{Sasakian manifolds}

Let $(X, g)$ be a connected oriented smooth Riemannian manifold of dimension $2 n+1$, where $n$ is a nonnegative integer. Denote by $\nabla$ the Levi-Civita connection. We recall the following definition.

Definition 2.1 ([4, Definition-Theorem 10]). The pair $(X, g)$ is called a Sasakian manifold if any of the following three equivalent conditions hold:

(i) There is a Killing vector field $\xi$ on $X$ of unit length such that the section

$$
\Phi \in C^{\infty}\left(X, \mathrm{TX} \otimes(\mathrm{TX})^{*}\right)
$$

defined by $\Phi(v)=-\nabla_{v} \xi$ satisfies the identity

$$
\left(\nabla_{v} \Phi\right)(w)=g(v, w) \xi-g(\xi, w) v
$$

for all $v, w \in T_{x} X$ and all $x \in X$.

(ii) There is a Killing vector field $\xi$ on $X$ of unit length such that the Riemann curvature tensor $R$ of $(X, g)$ satisfies the identity

$$
R(v, \xi) w=g(\xi, w) v-g(v, w) \xi
$$

for all $v$ and $w$ as above.

(iii) The metric cone $\left(\mathbb{R}_{+} \times X, d r^{2} \oplus r^{2} g\right)$ is Kähler. 
A Killing vector field $\xi$ on $X$ of unit length satisfies condition (i), if and only if it satisfies condition (ii). Given a Killing vector field $\xi$ of unit length satisfying condition (i), the Kähler structure on $\mathbb{R}_{+} \times X$ asserted in statement (iii) is constructed as follows. Let $F$ be the distribution of $X$ of rank $2 n$ given by the orthogonal complement of $\xi$. The homomorphism $\Phi$ (defined in (2.1)) preserves $F$, and furthermore,

$$
\left(\left.\Phi\right|_{F}\right)^{2}=-\operatorname{Id}_{F} .
$$

Let $\widetilde{J}$ be the almost complex structure on $\mathbb{R}_{+} \times X$ defined by the following conditions:

$$
\begin{gathered}
\left.\widetilde{J}\right|_{F}=\left.\Phi\right|_{F} \\
\widetilde{J}\left(\frac{d}{d r}\right)=\xi \\
\widetilde{J}(\xi)=-\frac{d}{d r} .
\end{gathered}
$$

This almost complex structure is integrable. The Riemannian metric $d r^{2} \oplus r^{2} g$ on $\mathbb{R}_{+} \times X$ is Kähler with respect to the complex structure $\widetilde{J}$.

Conversely, if the metric cone $\left(\mathbb{R}_{+} \times X, d r^{2} \oplus r^{2} g\right)$ is Kähler, then consider the vector field on $\mathbb{R}_{+} \times X$ given by $J(d / d r)$, where $J$ is the almost complex structure on $\mathbb{R}_{+} \times X$. The vector field $\xi$ on $X$ obtained by restricting this vector field to $\{1\} \times X=X$ satisfies condition $(i)$, and hence condition (ii), in Definition 2.1.

We will consider the vector field $\xi$ (or equivalently, the Kähler structure on $\left.\mathbb{R}_{+} \times X\right)$ as part of the definition of a Sasakian manifold.

Let $X$ be a smooth oriented Riemannian manifold of dimension $2 n+1$ and $F \subset T X$ an oriented smooth distribution of rank $2 n$. The quotient map

$$
\mathrm{TX} \longrightarrow \mathrm{TX} / F=: N
$$

defines a smooth one form on $X$

$$
\omega \in C^{\infty}\left(X, T^{*} X \otimes N\right)
$$

with values in the line bundle $N$. Since $X$ is oriented, the orientation of $F$ induces an orientation of the normal bundle $N$. Therefore, $N$ has a canonical smooth section given by the positively oriented vectors of unit length in the fibers of $N$. Consequently, the form $\omega$ in (2.7) gives a nowhere vanishing smooth one form on $X$. This one form will also be denoted by $\omega$. The 
distribution $F$ is said to be a contact structure on $X$ if the $(2 n+1)$-form $(d \omega)^{n} \wedge \omega$ is nowhere vanishing.

Remark 2.1. The distribution $F$ is integrable, if it satisfies the Frobenius condition which says that the one-forms $\omega$ satisfy the condition $(d \omega) \wedge \omega=0$. Therefore, a contact structure $F$ is not integrable unless $n=0$.

Now let $(X, g, \xi)$ be a Sasakian manifold. The distribution $F$ on $X$ of rank $2 n$ given by the orthogonal complement of the Killing vector field $\xi$ defines a contact structure on $X$. We note that the corresponding one-form $\omega$ is the dual of $\xi$ with respect to the metric $g$. From the condition that $(d \omega)^{n} \wedge \omega$ is nowhere vanishing it follows that the restriction of $d \omega$ to $F$ is fiberwise nondegenerate.

Lemma 2.1. For all $x \in X$ and all $v, w \in F_{x}$,

$$
d \omega(v, w)=-g(\Phi(v), w),
$$

where $\Phi$ is defined in (2.1).

Proof. From the definition of $\Phi$,

$$
-g(\Phi(v), w)=g\left(\nabla_{v} \xi, w\right) .
$$

Since $\xi$ is a Killing vector field,

$$
g\left(\nabla_{v} \xi, w\right)+g\left(\nabla_{w} \xi, v\right)=0 .
$$

Extend $v$ and $w$ to smooth sections $\widetilde{v}$ and $\widetilde{w}$ of $F$. Since $\widetilde{w}$ is orthogonal to $\xi$,

$$
g\left(\nabla_{v} \xi, w\right)=-g\left(\xi, \nabla_{v} \widetilde{w}\right)
$$

Using (2.9),

$$
g\left(\nabla_{v} \xi, w\right)=-g\left(\nabla_{w} \xi, v\right)=g\left(\xi, \nabla_{w} \widetilde{v}\right)
$$

because $\widetilde{v}$ is also orthogonal to $\xi$. Therefore,

$$
-g(\Phi(v), w)=\frac{1}{2}\left(-g\left(\xi, \nabla_{v} \widetilde{w}\right)+g\left(\xi, \nabla_{w} \widetilde{v}\right)\right)=-\frac{1}{2} g(\xi,[\widetilde{v}, \widetilde{w}]) .
$$

But $\left.-\frac{1}{2} g(\xi, \widetilde{v}, \widetilde{w}]\right)=d \omega(v, w)$ because both $\widetilde{v}$ and $\widetilde{w}$ are orthogonal to $\xi$. 
The line subbundle of $T X$ generated by $\xi$ will be denoted by $N$.

A compact connected Sasakian manifold $(X, g, \xi)$ is called quasi-regular if all the orbits of the unit vector field $\xi$ are closed.

\section{Holomorphic vector bundles}

\subsection{Differential forms and Hodge type decomposition}

Let $(X, g, \xi)$ be a Sasakian manifold. Let $E$ be a $C^{\infty}$ complex vector bundle over $X$.

Definition 3.1. A locally defined complex differential form $\alpha$ of class $C^{\infty}$ on $X$ will be called transversal if

$$
i_{\xi} \alpha=0 \quad \text { and } \quad i_{\xi} d \alpha=0
$$

where $i_{\xi}$ is the contraction with the Killing vector field $\xi$.

Since $d^{2}=0$, it follows immediately that for any transversal differential form $\alpha$ also $d \alpha$ is transversal.

Let $L_{\xi}$ denote the Lie derivative with respect to $\xi$. Since

$$
L_{\xi} \alpha=d i_{\xi} \alpha+i_{\xi} d \alpha,
$$

we conclude that $\alpha$ is transversal, if and only if

$$
i_{\xi} \alpha=0=L_{\xi} \alpha
$$

Let $\alpha$ be a locally defined transversal complex $d$-form. Take any point $x \in X$. Since $i_{\xi} \alpha=0$, the evaluation of $\alpha(x)$ on $\bigwedge^{d} T_{x} X \otimes_{\mathbb{R}} \mathbb{C}$ is determined by the evaluation of $\alpha(x)$ on the subspace

$$
\bigwedge^{d} F_{x} \otimes_{\mathbb{R}} \mathbb{C} \subset \bigwedge^{d} T_{x} X \otimes_{\mathbb{R}} \mathbb{C}
$$

where $F_{x}$ as before is the orthogonal complement of the line $\xi(x) \subset T_{x} X$. 
We recall that

$$
\left(\left.\Phi\right|_{F_{x}}\right)^{2}=-\operatorname{Id}_{F_{x}}
$$

(see (2.3)). Let

$$
\Phi_{x}^{\mathbb{C}}:=\left.\Phi\right|_{F_{x}} \otimes_{\mathbb{R}} \mathbb{C}: F_{x} \otimes_{\mathbb{R}} \mathbb{C} \longrightarrow F_{x} \otimes_{\mathbb{R}} \mathbb{C}
$$

be the complexification of the automorphism $\left.\Phi\right|_{F_{x}}$. From (3.1) it follows that the eigenvalues of $\Phi_{x}^{\mathbb{C}}$ are $\pm \sqrt{-1}$. We have a decomposition

$$
F_{x} \otimes_{\mathbb{R}} \mathbb{C}=F_{x}^{1,0} \oplus F_{x}^{0,1},
$$

where $F_{x}^{1,0}$ and $F_{x}^{0,1}$ are the eigenspaces for the eigenvalues $\sqrt{-1}$ and $-\sqrt{-1}$, respectively.

Now, for $p, q \geq 0$, define

$$
F_{x}^{p, q}:=\left(\bigwedge^{p} F_{x}^{1,0}\right) \otimes\left(\bigwedge^{q} F_{x}^{0,1}\right) \subset \bigwedge^{p+q} F_{x} \otimes_{\mathbb{R}} \mathbb{C} .
$$

Therefore, we have a decomposition

$$
\bigwedge^{d} F_{x} \otimes_{\mathbb{R}} \mathbb{C}=\bigoplus_{i=0}^{d} F_{x}^{i, d-i}
$$

for all $d \geq 0$.

Take a transversal complex $d$-form $\alpha$ defined over an open subset $U \subset X$ such that $\alpha$ is nonzero at some point. We will say that $\alpha$ is of type $(a, d-a)$, if the evaluation of $\alpha$ on $F_{x}^{i, d-i}$ vanishes for all $i \neq a$ and all $x \in U$.

Let $F^{i, d-i}$ denote the $C^{\infty}$ subbundle of the vector bundle $\bigwedge^{d} F \otimes_{\mathbb{R}} \mathbb{C}$ defined by the condition that the fiber of $F^{i, d-i}$ over each point $x \in X$ is $F_{x}^{i, d-i}$. The pointwise decomposition in (3.2) gives the following $C^{\infty}$ decomposition into a direct sum of vector bundles

$$
\bigwedge^{d} F \otimes_{\mathbb{R}} \mathbb{C}=\bigoplus_{i=0}^{d} F^{i, d-i}
$$

Now the decomposition $T X=F \oplus(\mathbb{R} \cdot \xi)$ induces a decomposition

$$
\bigwedge^{d} T X \otimes_{\mathbb{R}} \mathbb{C}=\bigwedge^{d} F_{x} \otimes_{\mathbb{R}} \mathbb{C} \oplus\left(\xi \otimes \bigwedge^{d-1} F_{x} \otimes_{\mathbb{R}} \mathbb{C}\right)
$$


for each $d \geq 0$. Combining this decomposition with the decomposition in (3.3), we have

$$
\bigwedge^{d} T X \otimes_{\mathbb{R}} \mathbb{C}=\bigoplus_{i=0}^{d} F^{i, d-i} \oplus\left(\xi \otimes\left(\bigoplus_{j=0}^{d-1} F^{j, d-j-1}\right)\right) .
$$

Consider the projection

$$
\bigwedge^{d} T X \otimes_{\mathbb{R}} \mathbb{C} \longrightarrow F^{i, d-i}
$$

obtained from the decomposition in (3.4). Its dual is an injective homomorphism

$$
f_{d, i}:\left(F^{i, d-i}\right)^{*} \longrightarrow \bigwedge^{d} T^{*} X \otimes_{\mathbb{R}} \mathbb{C}
$$

for each $d \geq 0$ and $i \leq d$. A nonzero transversal complex $d$-form $\alpha$ is of type $(a, d-a)$, if and only if $\alpha$ is of the form $f_{d, a}\left(\alpha^{\prime}\right)$ for some section $\alpha^{\prime}$ of $\left(F^{a, d-a}\right)^{*}$, where $f_{d, a}$ is the injective homomorphism in (3.5).

For any $i$ with $0 \leq i \leq d$, consider the inclusion of $F^{i, d-i} \hookrightarrow \bigwedge^{d} T X \otimes_{\mathbb{R}} \mathbb{C}$ in (3.4). Its dual is a projection

$$
\phi_{d, i}: \bigwedge^{d} T^{*} X \otimes_{\mathbb{R}} \mathbb{C} \longrightarrow\left(F^{i, d-i}\right)^{*} .
$$

Let $\alpha$ be a transversal differential form on $X$ of degree $d$. Take any $i$ with $0 \leq i \leq d$. Let

$$
\alpha_{i}:=f_{d, i} \circ \phi_{d, i}(\alpha)
$$

be the differential form on $X$, where $f_{d, i}$ and $\phi_{d, i}$ is constructed in (3.5) and (3.6), respectively.

Lemma 3.1. The differential form $\alpha_{i}$ in (3.7) is transversal.

Proof. From the construction of $\alpha_{i}$ is follows immediately that $i_{\xi} \alpha_{i}=0$.

To prove that $L_{\xi} \alpha_{i}=0$, consider the identity in (2.2). In it, set $v=\xi$, and set $w$ to be a smooth section of $F=\xi^{\perp}$. The identity in (2.2) immediately 
implies that

$$
\left(\nabla_{\xi} \Phi\right)(w)=0 .
$$

The automorphism $\Phi$ acts on $T^{*} X \otimes_{\mathbb{R}} \mathbb{C}$. From (3.8) it follows that the bundles consisting of eigenvectors associated to the action of $\Phi$ are preserved by $\xi$. In particular,

$$
L_{\xi}\left(f_{1,0} \circ \phi_{1,0}\right)=0=L_{\xi}\left(f_{1,1} \circ \phi_{1,1}\right)
$$

because $f_{1,0} \circ \phi_{1,0}$ and $f_{1,1} \circ \phi_{1,1}$ are projections to the eigenbundles for the eigenvalues $\sqrt{-1}$ and $-\sqrt{-1}$, respectively. Since $f_{d, j} \circ \phi_{d, j}$ are constructed from $f_{1,0} \circ \phi_{1,0}$ and $f_{1,1} \circ \phi_{1,1}$, we now have,

$$
L_{\xi}\left(f_{d, j} \circ \phi_{d, j}\right)=0
$$

for all $j$. In other words, the decomposition

$$
\mathrm{Id}=\bigoplus_{j=1}^{d} f_{d, j} \circ \phi_{d, j}
$$

is preserved by the flow on $X$ defined by $\xi$.

From (3.9) we have

$$
L_{\xi}\left(f_{d, i} \circ \phi_{d, i}(\alpha)\right)=f_{d, i} \circ \phi_{d, i}\left(L_{\xi} \alpha\right) .
$$

But $L_{\xi} \alpha=0$, because $\alpha$ is transversal. This completes the proof of the lemma.

Using the trivialization of $N$ given by $\xi$, the $N$-valued one-form $\omega$ becomes a real-valued one form on $X$. Now we consider the nowhere degenerate form $d \omega \mid F$. Using the orthogonal projection $T X \longrightarrow F$, this defines a two form on $X$ which is $d$-closed, but not exact in general. Then form $d \omega \mid F$ is clearly transversal. Now Lemma 2.1 has the following corollary:

Corollary 3.1. The transversal form $d \omega \mid F$ is of type $(1,1)$.

The formal adjoint of the multiplication operator

$$
\begin{aligned}
L:\left(F^{p, q}\right)^{*} & \longrightarrow\left(F^{p+1, q+1}\right)^{*} \\
\eta & \longmapsto \eta \wedge(d \omega \mid F)
\end{aligned}
$$

is denoted by

$$
\Lambda_{\omega}:\left(F^{p+1, q+1}\right)^{*} \longrightarrow\left(F^{p, q}\right)^{*} .
$$




\subsection{Partial connections}

Let

$$
S \subset T X \otimes_{\mathbb{R}} \mathbb{C}
$$

be a subbundle of positive rank, which is integrable. In other words, smooth sections of $S$ are closed under the operation of the Lie bracket. Let $E$ be a $C^{\infty}$ complex vector bundle over $X$. Let

$$
q_{S}: T^{*} X \otimes_{\mathbb{R}} \mathbb{C}=\left(T X \otimes_{\mathbb{R}} \mathbb{C}\right)^{*} \longrightarrow S^{*}
$$

be the dual of the inclusion map of $S$ in $T X \otimes_{\mathbb{R}} \mathbb{C}$.

A partial connection on $E$ in the direction of $S$ is a $C^{\infty}$ differential operator

$$
D: E \longrightarrow S^{*} \otimes E
$$

satisfying the Leibniz condition. The Leibniz condition says that for a smooth section $s$ of $E$ and a smooth function $f$ on $X$,

$$
D(f s)=f D(s)+q_{S}(d f) \otimes s
$$

holds, where $q_{S}$ is the projection in (3.12).

The condition that $D$ satisfies the Leibniz condition immediately implies that the order of the differential operator $D$ is exactly one. We recall that the symbol of a $C^{\infty}$ differential operator

$$
D^{\prime}: A \longrightarrow B
$$

of order one is a $C^{\infty}$ section of $A^{*} \otimes B \otimes\left(T X \otimes_{\mathbb{R}} \mathbb{C}\right)$.

Let $D$ be a partial connection on $E$ in the direction of $S$. The symbol of the first-order differential operator $D$ coincides with $\operatorname{Id}_{E} \otimes \widehat{q}_{S}$, where $\widehat{q}_{S}$ is the smooth section of $S^{*} \otimes T X \otimes_{\mathbb{R}} \mathbb{C}$ given by $q_{S}$ in (3.12). Indeed, this follows immediately from the fact that $D$ satisfies the Leibniz identity.

Since the distribution $S$ is integrable, smooth sections of $\operatorname{ker}\left(q_{S}\right)$ are closed under the exterior derivation. Therefore, we have an induced exterior derivation on the smooth sections of $S^{*}$

$$
\widehat{d}: S^{*} \longrightarrow \bigwedge^{2} S^{*}
$$

which is a differential operator of order one. 
Let $D$ be a partial connection on $E$ in the direction of $S$. Consider the differential operator

$$
D_{1}: S^{*} \otimes E \longrightarrow\left(\bigwedge^{2} S^{*}\right) \otimes E
$$

defined by

$$
D_{1}(\theta \otimes s)=\widehat{d}(\theta) \otimes s-\theta \wedge D(s),
$$

where $\widehat{d}$ is constructed in (3.14). The composition

$$
E \stackrel{D}{\longrightarrow} S^{*} \otimes E \stackrel{D_{1}}{\longrightarrow}\left(\bigwedge^{2} S^{*}\right) \otimes E
$$

is $C^{\infty}(X)$-linear. Therefore, the composition in (3.15) defines a $C^{\infty}$ section

$$
\mathcal{K}(D)=C^{\infty}\left(X,\left(\bigwedge^{2} S^{*}\right) \otimes E \otimes E^{*}\right)=C^{\infty}\left(X,\left(\bigwedge^{2} S^{*}\right) \otimes \operatorname{End}(E)\right) .
$$

The section $\mathcal{K}(D)$ in (3.16) is called the curvature of $D$. If

$$
\mathcal{K}(D)=0,
$$

then the partial connection $D$ is called flat.

\subsection{Holomorphic hermitian vector bundles}

Let $(X, g, \xi)$ be a Sasakian manifold. Let

$$
\widetilde{F}^{0,1}:=F^{0,1} \oplus\left(\xi \otimes_{\mathbb{R}} \mathbb{C}\right) \subset \mathrm{TX} \otimes_{\mathbb{R}} \mathbb{C}
$$

be the distribution, where $F^{0,1}$ is constructed in (3.3).

Lemma 3.2. The distribution $\widetilde{F}^{0,1}$ in (3.17) is integrable.

Proof. Let $v$ and $w$ be smooth sections of $F$. Then from (2.2),

$$
\left(\nabla_{v} \Phi\right)(w)=g(v, w) \xi
$$

In particular, this is a section of $\widetilde{F}^{0,1}$. Using this and (3.8) it follows that the distribution $\widetilde{F}^{0,1}$ is integrable. 
Definition 3.2. A Sasakian complex vector bundle on the Sasakian manifold $(X, g, \xi)$ is a pair $\left(E, D_{0}\right)$, where $E$ is a $C^{\infty}$ complex vector bundle on $X$, and $D_{0}$ is a partial connection in the direction $\xi$.

Since $N$ is a foliation on $X$ of dimension one, any partial connection in the direction $N$ is automatically flat.

Note that the vector field $\xi$ is contained in the foliation distribution $\widetilde{F}^{0,1}$ in (3.17). Therefore, any partial connection $D$ on $E$ in the direction of $\widetilde{F}^{0,1}$ defines a partial connection on $E$ in the direction of $N$.

Definition 3.3. A holomorphic vector bundle on the Sasakian manifold $(X, g, \xi)$ is a pair $\left(\left\{E, D_{0}\right\}, D\right)$, where $\left(E, D_{0}\right)$ is a $C^{\infty}$ Sasakian complex vector bundle on $X$, and $D$ is a flat partial connection on $E$ in the direction of $\widetilde{F}^{0,1}$ (constructed in (3.17)) satisfying the compatibility condition that $D_{0}$ coincides with the partial connection on $E$, in the direction of $\xi$, defined by $D$.

Definition 3.4. Let $\left(\left\{E, D_{0}\right\}, D_{E}\right)$ and $\left(\left\{E^{\prime}, D_{0}^{\prime}\right\}, D_{E^{\prime}}\right)$ be two holomorphic vector bundles on $(X, g, \xi)$. A fiberwise $\mathbb{C}$-linear $C^{\infty}$ map

$$
\Psi: E^{\prime} \longrightarrow E^{\prime \prime}
$$

is called holomorphic, if $\Psi$ intertwines $D_{E}$ and $D_{E^{\prime}}$.

Remark 3.1. Let $\left(\left\{E, D_{0}\right\}, D\right)$ be a Sasakian holomorphic vector bundle on $X$. Then the dual $E^{*}$ also has a natural structure of a Sasakian holomorphic vector bundle. If $\left(\left\{E^{\prime}, D_{0}^{\prime}\right\}, D^{\prime}\right)$ is another Sasakian holomorphic vector bundle, then $E \otimes E^{\prime}$ also has the structure of a Sasakian holomorphic vector bundle. In particular, $\operatorname{End}(E):=E \otimes E^{*}$ carries a natural structure of a Sasakian holomorphic vector bundle.

Definition 3.5. A hermitian structure on a Sasakian complex vector bundle $\left(E, D_{0}\right)$ is a $C^{\infty}$ hermitian structure on the complex vector bundle $E$ preserved by the partial connection $D_{0}$.

So a hermitian structure on a Sasakian holomorphic vector bundle induces a hermitian structure on the dual Sasakian holomorphic vector bundle. Similarly, hermitian structures on two Sasakian holomorphic vector bundles induce a hermitian structure on their tensor product.

Let $\left(\left\{E, D_{0}\right\}, D\right)$ be a Sasakian holomorphic vector bundle on $X$ equipped with a hermitian metric $h$. Let $D^{\prime}$ be a connection on $E$ preserving $h$ such that the partial connection on $E$ in the direction of $\xi$ induced by $D^{\prime}$ coincides 
with $D_{0}$. Let $s$ and $t$ be two locally defined smooth sections of $E$ which are flat with respect to the partial connection $D_{0}$. We have

$$
\phi_{1,1}(d(h(s, t)))=h\left(\phi_{1,1}\left(D^{\prime}(s)\right), t\right)+h\left(s, \phi_{1,1}\left(D^{\prime}(t)\right)\right),
$$

where $\phi_{i, j}$ is defined in (3.6). Since

$$
F \otimes_{\mathbb{R}} \mathbb{C}=F^{0,1} \oplus F^{1,0}=F^{0,1} \oplus \overline{F^{0,1}},
$$

it follows from (3.18) that there is a unique connection $\nabla$ on the complex vector bundle $E$ satisfying the following two conditions:

- $\nabla$ preserves $h$, and

- the partial connection on $E$ in the direction of $\widetilde{F}^{0,1}$ induced by $\nabla$ coincides with $D$.

Let $\mathcal{K}(E, h):=\mathcal{K}(\nabla)$ be the curvature of the above-mentioned connection $\nabla$. We observe that $\mathcal{K}(D)$ is a section of $\left(F^{1,1}\right)^{*} \otimes E \otimes E^{*}$.

The Chern forms $c_{j}(E, h)$ of the Sasakian holomorphic hermitian vector bundle are defined using $\mathcal{K}(E, h)$ in the usual way. More precisely,

$$
\operatorname{det}\left(\operatorname{id}_{E}+\frac{\sqrt{-1}}{2 \pi} \mathcal{K}(E, h)\right)=\sum_{i \geq 0} c_{i}(E, h) .
$$

We note that each $c_{i}(E, h)$ is a closed form of degree $i$, and $c_{i}(E, h)$ is of type $(i, i)$.

Henceforth, all Sasakian manifold considered will be assumed to be compact.

Definition 3.6. A Sasakian holomorphic, hermitian vector bundle $(E, h)$ is called Hermite-Einstein, if

$$
\sqrt{-1} \Lambda_{\omega} \mathcal{K}(E, h)=\lambda \operatorname{id}_{E}
$$

for some constant real number $\lambda$. 
The constant $\lambda$ is determined a priori. We define

$$
\operatorname{vol}(X):=\int_{X}(d \omega)^{n} \wedge \omega
$$

Then

$$
\frac{1}{2 \pi} \lambda \mathrm{rk} E \operatorname{vol}(E)=\int_{X} c_{1}(E, h)(d \omega)^{n} \wedge \omega=: \operatorname{deg}_{\omega}(E, h),
$$

where $\operatorname{dim} X=2 n+1$, is actually independent of the choice of $h$.

\subsection{Stable Sasakian holomorphic vector bundles}

We are in a position to define the stability of a Sasakian holomorphic vector bundle with respect to the contact form $\omega$. Stability is defined in terms of coherent subsheaves. We first define the structure sheaf $\mathcal{O}_{X}$ to be the sheaf of smooth functions $f$ with the property that $d f$ lies in the image of $f_{1,1}$, where $f_{1,1}$ is constructed in (3.5).

Definition 3.7. A coherent sheaf on a Sasakian manifold $X$ is a sheaf of $\mathcal{O}_{X}$-modules, which is locally the cokernel of a morphism of Sasakian vector bundles of the form

$$
\mathcal{O}_{X}^{\oplus a} \longrightarrow \mathcal{O}_{X}^{\oplus b}
$$

(see Definition 3.4).

Given a coherent Sasakian sheaf $\mathcal{E}$, there is a Sasakian holomorphic line bundle $\operatorname{det}(\mathcal{E})$ associated to it. Given any local resolution

$$
0 \longrightarrow V_{n} \longrightarrow V_{n-1} \longrightarrow \cdots \longrightarrow V_{0} \longrightarrow \mathcal{E} \longrightarrow 0
$$

over some open subset $U$, where each $V_{i}$ is a Sasakian holomorphic vector bundle for, and all the homomorphisms are morphisms of Sasakian vector bundles, the restriction of $\operatorname{det}(\mathcal{E})$ to $U$ is identified with $\otimes_{i=0}^{n} \operatorname{det}\left(V_{i}\right)^{(-1)^{i}}$. These locally defined line bundles patch together in a natural way to define the line bundle $\operatorname{det}(\mathcal{E})$ over $X$.

The degree $\operatorname{deg} \mathcal{E}$ of a torsionfree coherent Sasakian sheaf is defined to be

$$
\operatorname{deg} \mathcal{E}:=\int_{X} c_{1}(\operatorname{det}(\mathcal{E}))(d \omega)^{n-1} \wedge \omega
$$

(recall that $X$ is compact). 
Definition 3.8. Let $E=\left(\left\{E, D_{0}\right\}, D\right)$ be a Sasakian holomorphic vector bundle, and $\mathcal{E}$ the associated locally free $\mathcal{O}_{X}$-module. The bundle $E$ is called stable if for any Sasakian coherent subsheaf $\mathcal{E}^{\prime} \subset \mathcal{E}$ such that the quotient $\mathcal{E} / \mathcal{E}^{\prime \prime}$ is torsionfree of positive rank, the inequality

$$
\frac{\operatorname{deg}\left(\mathcal{E}^{\prime}\right)}{\operatorname{rk}\left(\mathcal{E}^{\prime}\right)}<\frac{\operatorname{deg}(\mathcal{E})}{\operatorname{rk}(\mathcal{E})}
$$

holds.

The bundle $E$ is called semistable, if

$$
\frac{\operatorname{deg}\left(\mathcal{E}^{\prime}\right)}{\operatorname{rk}\left(\mathcal{E}^{\prime}\right)} \leq \frac{\operatorname{deg}(\mathcal{E})}{\operatorname{rk}(\mathcal{E})} .
$$

\subsection{Sasakian Hermite-Einstein metrics and applications}

Let $(X, g, \xi)$ be a quasi-regular Sasakian manifold. From now onwards we will assume quasi-regularity.

Recall that $F=N^{\perp} \subset \mathrm{TX}$ is the orthogonal complement of the onedimensional foliation generated by the Killing vector field $\xi$. We will call $F$ the horizontal distribution. In (2.3) we noted that $\Phi$ preserves the horizontal distribution.

The closedness of the $\xi$-orbits amounts to an action of the circle group

$$
\mathbb{S}^{1}=\{z \in \mathbb{C}|| z \mid=1\}
$$

on $X$. However, the non-integrability of the contact structure defined by the horizontal distribution $F$ means that we can just consider local slices transversal to $\xi$, which defines a complex orbifold structure on the quotient $X / \mathbb{S}^{1}$. The Sasakian holomorphic structure for complex vector bundles discussed in Section 3.3 now can be studied locally. This is actually sufficient to show the existence of Hermite-Einstein metrics on stable bundles.

Existence of Hermite-Einstein structures. Let $(X, g, \xi)$ be a compact quasi-regular Sasakian manifold, and let $\left(\left\{E, D_{0}\right\}, D\right)$ be a stable Sasakian holomorphic vector bundle on it. Then there exists a HermiteEinstein structure on E. Furthermore, the corresponding connection is uniquely determined.

We indicate, why the now standard methods of Donaldson [5] and Uhlenbeck and Yau [9] are applicable. 
Given an initial hermitian metric $h_{0}$ on $E$ in the sense of Definition 3.5, we consider the heat equation for a one-parameter family $\left\{h_{t}\right\}_{t \geq 0}$ of Sasakian hermitian metrics with curvature $\mathcal{K}\left(h_{t}\right)$

$$
\frac{\partial h_{t}}{\partial t} h_{t}^{-1}=\Lambda_{\omega} \sqrt{-1} \mathcal{K}\left(h_{t}\right)-\lambda \operatorname{id}_{\mathrm{E}} .
$$

This equation is not parabolic on $X$. We note that the two-form $\left.(d \omega)\right|_{F}$ as well as the Riemannian metric $g$ are $\mathbb{S}^{1}$-invariant. Hence, the contraction operation $\Lambda_{\omega}$ in (3.10) is invariant under the action of $\mathbb{S}^{1}$. With the restriction on hermitian metrics in Definition 3.5, the above heat equation is $\mathbb{S}^{1}$-invariant, and it exists as a parabolic equation on any slice. The maximum principle is applicable because of the compactness of $X$. The usual $C^{0}$ - estimates for the pointwise norm of the curvature follow from

$$
\frac{d}{d t}\left|\Lambda_{\mathcal{K}}\left(h_{t}\right)\right|^{2}=\square\left|\Lambda_{\mathcal{K}}\left(h_{t}\right)\right|^{2}-\left|\nabla \Lambda_{\mathcal{K}}\left(h_{t}\right)\right|^{2},
$$

where $\square$ must be defined in the sense of the horizontal distribution $F$. The existence of solutions for all $t \geq 0$ is provided. It follows that

$$
\frac{d}{d t} \int_{X}\left|\Lambda_{\mathcal{K}}\left(h_{t}\right)\right|^{2}(t)=-\int_{X}\left|\nabla \Lambda_{\mathcal{K}}\left(h_{t}\right)\right|^{2}(t),
$$

and integration over $t$ yields the existence of a sequence $t_{j} \rightarrow \infty$ such that

$$
\int_{X}\left|\nabla \Lambda_{\mathcal{K}}\left(h_{t}\right)\right|^{2}\left(t_{j}\right) \longrightarrow 0
$$

The final step, following Uhlenbeck and Yau [9], of finding suitable gauge transformations such that the transformed hermitian metrics converge to a Sasakian Hermite-Einstein metric is based upon purely local arguments. In our case, it can be carried through on the local slices transversal to $\xi$.

Now we need the notion of a holomorphic family of holomorphic Sasakian vector bundles.

Let $(X, g, \xi)$ be a compact quasi-regular Sasakian manifold, and let $S$ be a parameter space, which will be a complex manifold (later we will also consider reduced complex spaces). We equip $S$ with the flat Kähler form. We extend the Killing field $\xi$ to a vector field on $X \times S$ using the decomposition of $T(X \times S)$, and denote it by $\xi_{S}$. Extend the horizontal distribution $F$ to a distribution $F_{S}$ on $X \times S$ of codimension one by taking the direct sum of 
$F$ with TS. Like in Section 3.1 we can now define $\Lambda^{p} F_{S}$ and $F_{S}^{p, q}$. As in (3.17) define

$$
\widetilde{F}_{S}^{0,1}:=F_{S}^{0,1} \oplus \xi_{S} \otimes_{\mathbb{R}} \mathbb{C}
$$

Definition 3.9. Let $(X, g, \xi)$ be a compact quasi-regular Sasakian manifold.

(i) A family $\left\{\left(\mathcal{E}_{s}, D_{0, s}\right)\right\}_{s \in S}$ of Sasakian complex vector bundles parameterized by $S$ is given by a complex vector bundle $\mathcal{E}$ of class $C^{\infty}$ over $X \times S$ together with a partial connection $D_{0, \mathcal{E}}$ in the direction of $\xi$. For $s \in S$, we define $\left(\mathcal{E}_{s}, D_{0, s}\right):=\left.\left(\mathcal{E}, D_{0, \mathcal{E}}\right)\right|_{X \times\{s\}}$.

(ii) A holomorphic family of Sasakian vector bundles is a family of Sasakian complex vector bundles $\left\{\left(\mathcal{E}_{s}, D_{0, s}\right)\right\}_{s \in S}$ (as in (i)) together with a flat partial connection $D$ on $\mathcal{E}$ in the direction on $\widetilde{F}_{S}^{0,1}$ (defined in (3.21)) satisfying the following condition: for all $s \in S$, the partial connection on $\mathcal{E}_{s}$ in the direction of $\xi$ defined by $D$ coincides with $D_{0, s}$. The restriction of $D$ to $\mathcal{E}_{s}$ will be denoted by $D_{s}$.

We observe that the complex orbifold structure on $X / \mathbb{S}^{1}$ guarantees that for holomorphic families of Sasakian holomorphic vector bundles, the Schlessinger conditions of deformation theory hold, in particular we have universal deformations for simple bundles rather than semi-universal ones. A Sasakian holomorphic vector bundle is called simple, if all of its automorphisms are constant scalar multiplications. (In the above setting, the base was assumed to be smooth. The usual approach would be to consider differentiable families of complex vector bundles first, and use the integrability condition for the holomorphic structures, which defines the base space $S$ as a subset of a smooth local ambient space, in the last step. In this way all relevant differential operators exist on the ambient space of the base [6].)

Theorem 3.1. Let $(X, g, \xi)$ be a compact quasi-regular Sasakian manifold. Then there exists the moduli space of stable, Sasakian holomorphic vector bundles on $X$ in the category of Hausdorff complex spaces.

Proof. Since $X$ is compact and quasi-regular, any section of $\mathcal{O}_{X}$ (defined earlier) is a constant function. Therefore, all the eigenfunctions of an endomorphism of a holomorphic Sasakian vector bundle on $X$ are constant functions. By this argument any stable Sasakian holomorphic vector bundle is simple. So the universal deformations of stable Sasakian holomorphic vector bundles exist. The local patching of the base spaces $S$ of universal deformations yields a (reduced) complex space. Over these we are given holomorphic families of Sasakian holomorphic vector bundles equipped with 
Hermite-Einstein metrics, which can be chosen to depend in a $C^{\infty}$ way on the parameter.

The Hausdorff property follows from the existence and uniqueness of Hermite-Einstein connections:

Let $S$ and $S^{\prime}$ be reduced complex spaces, and let $\left\{E_{s}, h_{s}\right\}_{s \in S}$ and $\left\{E_{s}^{\prime}, h_{s}^{\prime}\right\}_{s \in S^{\prime}}$ be families of Sasakian holomorphic Hermite-Einstein bundles. The functor

$$
I_{\operatorname{som}_{S \times S^{\prime}}}\left(E \times S^{\prime}, S \times E^{\prime}\right) \longrightarrow((\text { Sets })),
$$

which assigns to any space $R \longrightarrow S \times S^{\prime}$ the set of holomorphic isomorphisms of the bundles pulled back to $R$ (and whose morphisms are defined in the obvious way) is representable by a complex space

$$
\psi: I \longrightarrow S \times S^{\prime}
$$

together with a universal object. The fibers of $\psi$ are either empty or torsors for $\mathbb{C}^{*}$. Because of stability, $\psi$ defines a $\mathbb{C}^{*}$-bundle over its (closed analytic) image. Given the choice of relative Hermite-Einstein metrics for both families, the functor of isometries is represented by

$$
\psi^{\mathrm{HE}}: I^{\mathrm{HE}} \longrightarrow S \times S^{\prime}
$$

where $I \supset I^{\mathrm{HE}} \longrightarrow S \times S^{\prime}$ is a principal $\mathrm{U}(1)$-bundle. Let

$$
\Gamma=\left\{\left(s, s^{\prime}\right) \in S \times S^{\prime}, \mid E_{s} \sim E_{s^{\prime}}^{\prime} \text { isometrically equivalent }\right\} .
$$

For any $\left(s, s^{\prime}\right) \in S \times S^{\prime}$ the corresponding Hermite-Einstein metrics are gauge equivalent. Now

$$
\Gamma=\psi^{\mathrm{HE}}\left(I^{\mathrm{HE}}\right)=\psi(I)
$$

is a closed analytic subspace of $S \times S^{\prime}$, which shows the properness of the equivalence relation given by $\Gamma$.

Remark 3.2. Since the group of holomorphic isometries of HermiteEinstein bundles is connected, the moduli space is the union of deformation spaces of stable Sasakian bundles. 


\subsection{Sasakian moduli metric for vector bundles}

In this section we will introduce a Kähler structure on the moduli space of stable Sasakian holomorphic vector bundles, which is constructed using a certain determinant line bundle, equipped with a Quillen metric.

We first introduce the Sasakian moduli form. The construction will be functorial with respect to base change.

Let $\left.\left\{\left(\mathcal{E}_{s}, D_{0, s}\right\}, D_{s}\right)\right\}_{s \in S}$ be a holomorphic family of holomorphic Sasakian vector bundles on $(X, g, \xi)$, equipped with a $C^{\infty}$ family of Hermite-Einstein metrics $\left\{h_{s}\right\}$, amounting to a hermitian metric on the complex vector bundle $\left(\mathcal{E}, D_{0 \mathcal{E}}\right)$. Let $\mathcal{K}(\mathcal{E}, h)$ be the curvature form of the unique hermitian connection on $\mathcal{E}$ compatible with its holomorphic structure. Therefore, $\mathcal{K}(\mathcal{E}, h)$ is a smooth section of $\left(F_{S}^{1,1}\right)^{*} \otimes \operatorname{End}(\mathcal{E})$.

Definition 3.10. The Sasakian moduli form for vector bundles is defined as the following fiber integral:

$$
\omega^{\mathrm{SB}}=\int_{(X \times S) / S} \operatorname{tr}(\sqrt{-1} \mathcal{K}(\mathcal{E}, h) \wedge \sqrt{-1} \mathcal{K}(\mathcal{E}, h)) \frac{(d \omega)^{n}}{n !} \omega .
$$

It follows from its construction that the Sasakian moduli form is a $d$-closed real $(1,1)$-form on the parameter space $S$. Using the above arguments, one can see that it possesses, in the case where $S$ is reduced but possibly singular, a local $\partial \bar{\partial}$-potential of class $C^{\infty}$.

We know that the curvature form of a hermitian, Sasakian holomorphic vector bundle induces a real $(1,1)$-form, with values in the endomorphism bundle, in horizontal direction. The same holds for the curvature form of a hermitian vector bundle over $X \times S$, which defines a family of such objects. For this reason, we are in a position to carry over the methods from [3] literally to the orbifold case of Hermite-Einstein orbifold vector bundles over the orbifold $X / \mathbb{S}^{1}$ (cf. also [1]). We arrive at the following:

Theorem 3.2. The Sasakian moduli form for holomorphic vector bundles $\omega^{\mathrm{SB}}$ is a Kähler form on the moduli spaces of Sasakian holomorphic stable vector bundles. It possesses local $\partial \bar{\partial}$-potentials of class $C^{\infty}$ (with respect to ambient smooth spaces).

Let $M$ be a complex projective manifold and $L$ a very ample line bundle over $M$. Fix a positive hermitian structure on $L$. Let $\omega$ denote the Kähler 
form on $M$ given by its curvature. Define

$$
X:=\{v \in L \mid\|v\|=1\} .
$$

The group $\mathbb{S}^{1}$ has the natural multiplication action on $X$. The hermitian connection on $L$ gives a splitting of $T X$. We define a Riemannian structure on $X$ by assigning the $\omega$ in the horizontal direction; the metric in the vertical direction is given by the standard metric on $\mathbb{S}^{1}$. It is easy to see that the Riemannian manifold $X$ equipped with the vector field $\xi$ that is given by the action of $\mathbb{S}^{1}$, is a Sasakian manifold. We note that the embedding

$$
M \hookrightarrow P\left(H^{0}(M, L)^{*}\right)
$$

given by the linear system lifts to a diffeomorphism

$$
X \longrightarrow H^{0}(M, L)^{*}
$$

which is an isometry in the horizontal direction.

Definition 3.11. A Sasakian manifold $(X, g, \xi)$ is called of projective type if it possesses a holomorphic hermitian Sasakian line bundle $\left(L^{S}, h^{S}\right)$ whose Chern form is a positive hermitian form on $F^{1,0}$.

Now we construct the determinant line bundle $\lambda$ on the base of a universal deformation. The construction is based upon the Riemann-Roch formula for hermitian vector bundles by Bismut et al. [2], which also holds for orbifolds (cf. [7]).

Let $(\mathcal{E}, h)$ be a family of holomorphic hermitian vector bundles of rank $r$ over $X$ parameterized by $S$. Let

$$
\pi:\left(X / \mathbb{S}^{1}\right) \times S \longrightarrow X / \mathbb{S}^{1}
$$

be the projection. The following formula holds for Chern characters for elements in the Grothendieck's $K$-group:

$$
\begin{aligned}
\chi & :=\left(r^{2}-\operatorname{ch}\left(\mathcal{E} \otimes \mathcal{E}^{*}, h\right)\right) \cdot \operatorname{ch}\left(\left((L, h)-(L, h)^{-1}\right)^{\otimes(n-1)}\right) \\
& =2^{n-1} c_{1}^{2}(\mathcal{E}, h) c_{1}(L, h)^{n-1}+\text { higher order terms. }
\end{aligned}
$$

The $(1,1)$-component of the push forward $\pi_{*} \chi$ in $(3.23)$ is the $(1,1)$ component of the fiber integral, hence it is $\omega^{\mathrm{SB}}$ up to a numerical constant. 
In view of the Riemann-Roch formula, [2], the corresponding determinant line bundle is

$$
\lambda:=\operatorname{det} R^{\bullet} \pi_{*}\left(\left(\mathcal{O}_{X / \mathbb{S}^{1}}^{r^{2}}-\mathcal{E} \otimes \mathcal{E}^{-1}\right) \otimes\left(\pi^{*} L-\pi^{*} L^{-1}\right)^{\otimes(n-1)}\right) .
$$

We indicate, how the above determinant line bundle can be computed for a locally free sheaf $\mathcal{F}$ say and a proper holomorphic map $\pi: Z \longrightarrow S$ : Take the direct images $R^{q} \pi_{*} \mathcal{F}$ first. These locally possess free resolutions. Like in Section 3.4, we define a determinant line bundle associated to the resolution, which only depends upon the sheaf $R^{q} \pi_{*} \mathcal{F}$. Finally, the tensor power of these invertible sheaves with alternating exponent defines

$$
\lambda(\mathcal{F}):=\underline{\underline{\operatorname{det}}}(\mathcal{F}):=R^{\bullet} \pi_{*} \mathcal{F} .
$$

For a short exact sequence

$$
0 \longrightarrow \mathcal{F}^{\prime} \longrightarrow \mathcal{F} \longrightarrow \mathcal{F}^{\prime \prime} \longrightarrow 0,
$$

we have that $\underline{\operatorname{det}}(\mathcal{F})=\underline{\operatorname{det}}\left(\mathcal{F}^{\prime}\right) \otimes \underline{\operatorname{det}}\left(\mathcal{F}^{\prime \prime}\right)$. This fact allows for a generalization of the definition of determinant line bundles. If $\mathcal{F}$ denotes a coherent sheaf on $Z$, then $\mathcal{F}$ can be replaced by a locally free resolution in the algebraic case (and more generally by the corresponding simplicial objects in the non-algebraic case). Furthermore, the definition can be extended to elements of the relative $K$-groups. If we let $\mathcal{F}$ stand for an element of the relative $K$-group arising from hermitian vector bundles, like in the case under consideration, and $\pi: Z \rightarrow S$ is a smooth Kähler morphism with relative Kähler form induced by a closed form $\omega_{Z}$ on the total space, the main theorem of [2] states the existence of a Quillen metric $h^{Q}$ on $\lambda(\mathcal{F})$ such that its first Chern form equals

$$
c_{1}\left(\lambda(\mathcal{F}), h^{Q}\right)=-\left(\int_{Z / S} \operatorname{td}\left(Z / S, \omega_{Z}\right) \cdot \operatorname{ch}(\mathcal{F}, h)\right)_{(1,1)} .
$$

In this way $\lambda$ of (3.24) is being defined and equipped with a Quillen metric. It can be verified immediately that in our case the right-hand side of (3.25) is equal to the fiber integral of $\chi$, which was defined in (3.23). Now we have that the Chern form is up to a numerical constant equal to the Sasakian moduli metric form in the sense of orbifold structures. Observe that these arguments also hold in the case of an orbifold structure on a singular moduli space. The necessary techniques are in [6, Appendix]. 
Theorem 3.3. The Sasakian moduli metric form for vector bundles is up to a numerical factor equal to the Chern form of the determinant line bundle.

$$
c_{1}\left(\lambda, h^{Q}\right) \simeq \omega^{\mathrm{SB}} .
$$

\section{References}

[1] W. L. Baily, On the imbedding of $V$-manifolds in projective space, Amer. J. Math. 79 (1957), 403-430.

[2] J.-M. Bismut, H. Gillet and C. Soulé, Analytic torsion and holomorphic determinant bundles. I: Bott-Chern forms and analytic torsion; II: Direct images and Bott-Chern forms; III: Quillen metrics on holomorphic determinants, Commun. Math. Phys. 115 (1988), 49-78, 79-126, 301-351.

[3] I. Biswas and G. Schumacher, Determinant bundle, Quillen metric, and Petersson-Weil form on moduli spaces, Geom. Funct. Anal. 9 (1999), 226-255.

[4] C. P. Boyer and K. Galicki, Sasakian geometry, holonomy and supersymmetry, arXiv:math/0703231.

[5] S. K. Donaldson, Anti self-dual Yang-Mills connections over complex algebraic surfaces and stable vector bundles, Proc. London Math. Soc. 50 (1985), 1-26.

[6] A. Fujiki and G. Schumacher, The moduli space of extremal compact Kähler manifolds and generalized Weil-Petersson metrics. Publ. Res. Inst. Math. Sci. 26 (1990), 101-183.

[7] X. Ma, Orbifolds and analytic torsions, Trans. Amer. Math. Soc. 357 (2005), 2205-2233.

[8] J. Maldacena, The large $N$ limit of superconformal field theories and supergravity, Adv. Theor. Math. Phys. 2 (1998), 231-252.

[9] K. Uhlenbeck and S.-T. Yau, On the existence of Hermitian-Yang-Mills connections in stable vector bundles. Frontiers of the mathematical sciences, Comm. Pure Appl. Math. 39 (1986), S257-S293. 
